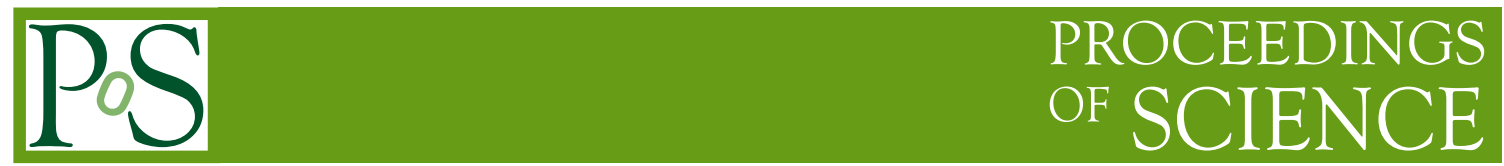

\title{
QCD at non-zero density and phenomenology
}

\author{
Claudia Ratti* \\ Physics Department, University of Houston \\ E-mail: cratti@uh.edu
}

\begin{abstract}
In the last few years, numerical simulations of QCD on the lattice have reached a new level of accuracy. A wide range of thermodynamic observables is now available in the continuum limit and for physical quark masses. This allows a quantitative comparison with measurements from heavy ion collisions. I will review the state-of-the-art results from lattice simulations of QCD thermodynamics and connect them to experimental measurements from RHIC, in view of the second Beam Energy Scan scheduled for 2019-2020.
\end{abstract}

The 36th Annual International Symposium on Lattice Field Theory - LATTICE2018 22-28 July, 2018

Michigan State University, East Lansing, Michigan, USA.

${ }^{*}$ Speaker. 


\section{Introduction}

The study of finite-density QCD aims at answering a series of fundamental questions in the next few years. In particular, the existence and location of a critical point separating the crossover at chemical potential $\mu_{B}=0$ [1] from a hypothetical first order phase transition, is one of the main open questions in our field. Other topics which will be addressed are the location of the transition line and the phases of QCD at high density. By systematically decreasing the collision energy, heavy ion collisions produce the high density phases of matter in the laboratory. The RHIC facility at BNL is particularly suited for this purpose: the second Beam Energy Scan is scheduled for 2019 and 2020. RHIC will run both in collider and fixed target modes, and will be able to reach high values of the baryonic chemical potentials. The study of dense matter will not stop after RHIC: NICA, CBM and JPARC will pursue the study of critical point, onset of deconfinement and dense hadronic matter at least till 2025.

Such a rich experimental program needs the support of fundamental theory and phenomenology. In this contribution I will focus on observables obtained from lattice QCD simulations, which are so precise to be employed in a quantitative comparison to experiments. These include the equation of state, which is needed as input of hydrodynamic codes which describe the evolution of matter created in heavy-ion collisions (see e.g. [2] and references therein); the information on the phase diagram and constraints on the critical point location; the fluctuations of conserved charges. The latter can be measured in experiments and in principle they allow a direct comparison between theory and heavy ion data, provided that non-thermal effects are understood and corrected for.

\section{Low-temperature phase: the Hadron Resonance Gas model}

In the low temperature phase, the results of lattice simulations for QCD thermodynamics are generally well described by the Hadron Resonance Gas (HRG) model, which has its roots in the theorem by Dashen, Ma and Bernstein [3]. This theorem allows one to calculate the microcanonical partition function of an interacting system, in the thermodynamic limit $V \rightarrow \infty$, assuming that it is a gas of non-interacting free hadrons and resonances [4]. This model needs as an input the list of all known baryons and mesons with their mass and quantum numbers. It was recently pointed out that additional resonances are needed in order to improve the agreement between lattice QCD and HRG model predictions [5, 6].

It is interesting to note that, setting $\mu_{S}=\mu_{Q}=0$, the dependence of the baryonic pressure on the baryonic chemical potential is extremely simple:

$$
\frac{p}{T^{4}}=\sum_{i \in B} \frac{d_{i}}{\pi^{2}}\left(\frac{m_{i}}{T}\right)^{2} \sum_{N=1}^{\infty}(-1)^{N+1} N^{-2} K_{2}\left(N \frac{m_{i}}{T}\right) \cosh \left[N \frac{\mu_{B}}{T}\right]
$$

any baryon contribution to the pressure is given by a function of the baryon mass $m_{i}$ and degeneracy $d_{i}$, times $\cosh \left[N \frac{\mu_{B}}{T}\right]$. The latter is the same for all baryons. The Boltzmann approximation corresponds to $N=1$ in the above fugacity expansion. If we now include a finite $\mu_{S}$ and $\mu_{Q}$, all baryons with the same strangeness and electric charge content will have the same factor, which contains the chemical potentials. This expansion can be used to separate the contribution of particles according to their quantum numbers. This idea, first developed in [5], has led to the identification 
of the missing resonance states according to their flavor content in Ref. [6]. We will come back to this point later, when discussing kaon fluctuations.

\section{QCD equation of state at zero and finite $\mu_{B}$}

The equation of state of QCD with $2+1$ flavors at $\mu_{B}=0$ is known for a few years. The WB collaboration published continuum extrapolated results at the physical quark mass based on a treelevel Symanzik improved gauge action with 2-step stout-link improved staggered fermions[7, 8, 9]; more recently, also the charm quark has been included in the system [10]. From this analysis, it turns out that the charm quark is a relevant degree of freedom already at $T \sim 250 \mathrm{MeV}$, which should be taken into account in hydrodynamic simulations of heavy ion collisions at the LHC energies [11]. The hotQCD collaboration found similar results [12] using the highly improved staggered quark (HISQ) action introduced in [13].
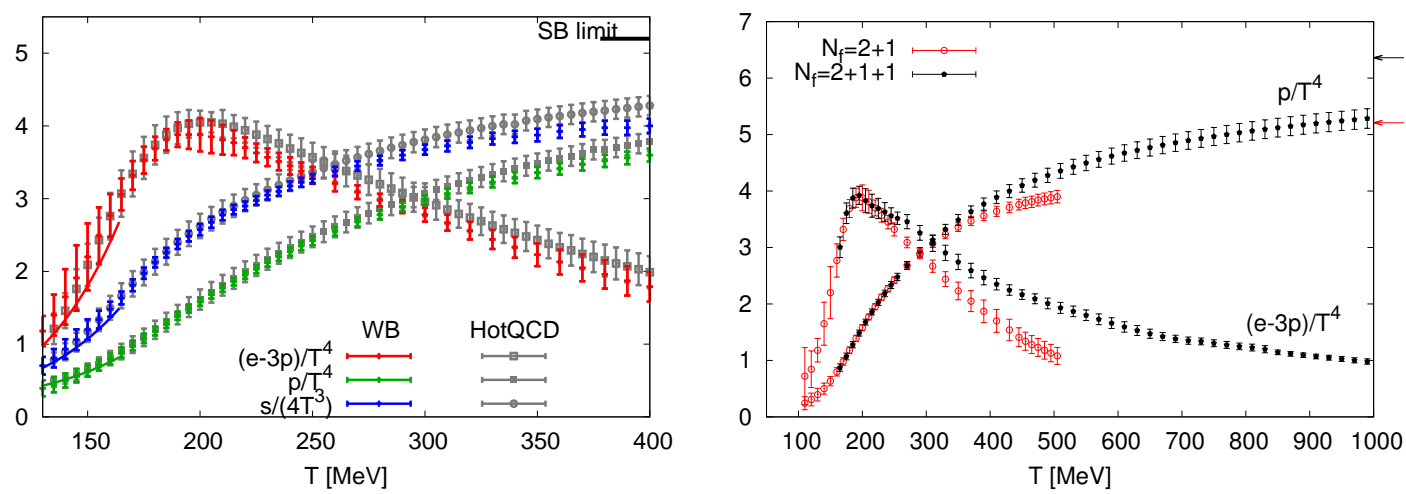

Figure 1: Left: Continuum extrapolated results for trace anomaly, entropy density and pressure. The gray points are from the HotQCD collaboration [12], while the colored ones are from the WB collaboration [9]. The figure also shows the Stefan-Boltzmann limit for the pressure and the scaled entropy; the curves at low temperature correspond to the HRG model predictions. Right: the trace anomaly and pressure in the $2+1$ and $2+1+1$ flavor theories (from Ref. [10]).

The left panel of Fig. 1 shows the comparison between the hotQCD (gray) and WB (colored) results for the trace anomaly, entropy density and pressure. The right panel is a comparison between the trace anomaly and pressure for a system of $2+1$ (red) and $2+1+1$ (black) dynamical quark flavors.

An important validation of the lattice QCD Equation of State has been obtained from a Bayesian analysis [14]. In this framework, a large number of observables from RHIC and the LHC has been compared to theoretical models, while varying the model parameters. The posterior distribution over possible equations of states turned out to be consistent with results from lattice QCD. This analysis has also been successfully applied to infer the behavior of other quantities, such as the shear viscosity of the QGP at zero [15] and finite density [16].

Direct simulation of QCD thermodynamics at finite density is not possible, due to the sign problem. Nevertheless, the need for finite- $\mu_{B}$ results to support the experimental program has driven the thermal lattice QCD community to find alternative approaches. Several methods have been proposed to calculate thermodynamics quantities at small chemical potential. These include 
Taylor expansion around $\mu_{B}=0[17,18,19,20,21]$, analytic continuation from imaginary $\mu_{B}$ $[22,23,23,24,25,26,27,28,29]$, reweighting of the generated configurations [30, 31, 32, 33], use of the canonical ensemble [34, 35, 36] and density of state methods [37, 38]. Here I will focus on the first two. The Taylor expansion for the pressure can be written as

$$
\frac{p\left(T, \mu_{B}\right)}{T^{4}}=\frac{p(T, 0)}{T^{4}}+\left.\sum_{n=1}^{\infty} \frac{1}{(2 n) !} \frac{d^{2 n}\left(p / T^{4}\right)}{d\left(\frac{\mu_{B}}{T}\right)^{2 n}}\right|_{\mu_{B}=0}\left(\frac{\mu_{B}}{T}\right)^{2 n}=\sum_{n=0}^{\infty} c_{2 n}(T)\left(\frac{\mu_{B}}{T}\right)^{2 n} .
$$

The Taylor coefficients can be calculated in two ways, either by direct simulations, or simulations at imaginary chemical potentials. Another important point is that, at finite $\mu_{B}$, one has to make choices also for the other two conserved charge chemical potentials, namely $\mu_{S}$ and $\mu_{Q}$. The two most popular choices are either $\mu_{S}=\mu_{Q}=0$, or $\mu_{S}$ and $\mu_{Q}$ functions of $T$ and $\mu_{B}$, such that we have $\left\langle n_{S}\right\rangle=0$ and $\left\langle n_{Q}\right\rangle=0.4\left\langle n_{B}\right\rangle$, to match the experimental situation in a heavy-ion collision.

More in detail, in the direct method a derivative of the partition function can be written in terms of the action with all fermionic degrees of freedom already integrated out, $S_{\text {eff }}$, as follows:

$$
\partial_{i} \log Z=\frac{1}{Z} \int \mathscr{D} U \partial_{i} e^{-S_{\text {eff }}}=\left\langle A_{i}\right\rangle .
$$

Here $i$ indicates the variable of the derivative, the chemical potential $\mu_{i}$ in this case. $A_{i}$ is the first derivative of $S_{\text {eff }}$ without the factor $e^{-S_{\text {eff }}}$. Its ensemble average is calculated with the same weight used for generating the configurations. In particular,

$$
A_{i}=\frac{1}{4} \operatorname{tr} M_{i}^{-1}\left(m_{i}, \mu_{i}\right) M_{i}^{\prime}\left(m_{i}, \mu_{i}\right),
$$

where $M_{i}\left(m_{i}, \mu_{i}\right)=m_{i}+\not \supset\left(\mu_{i}\right)$ is the fermion operator with the bare mass $m_{i} ; M_{i}^{\prime}\left(m_{i}, \mu_{i}\right)$ stands for its first derivative with respect to $\mu_{i}$. Higher order derivatives can be evaluated in a similar way. The most expensive part of this method is the calculation of the trace in Eq. (3.3), which contains disconnected contributions and appears in almost all susceptibilities.

After the early results for $c_{2}, c_{4}$ and $c_{6}$ [18], the first continuum extrapolated results for $c_{2}$ were published in Ref. [39]; in Ref. [40] $c_{4}$ was shown, but only at finite lattice spacing. The continuum limit for $c_{6}$ was published for the first time in [41] in the case of strangeness neutrality, and later in [42]. In the latter, both strangeness neutrality and $\mu_{S}=\mu_{Q}=0$ results are presented. A continuum estimate is performed, based on $N_{t}=6$ and 8 lattices. In [43], a first determination of $c_{8}$, at two values of the temperature and $N_{t}=8$ was presented. More recently, diagonal and non-diagonal coefficients up to $c_{8}$ have been calculated at $N_{t}=12$ in Ref. [44] at $\mu_{S}=\mu_{Q}=0$.

Figure 2 shows possible landscapes for simulations at imaginary $\mu_{B}$ and $\mu_{S}$. The black dot corresponds to direct simulation of all coefficients at $\mu_{B}=0$, as performed e.g. by the HotQCD collaboration. The red squares correspond to finite $\mu_{B}$ and $\mu_{S}=0$, while the green triangles are trajectories which ensure the strangeness-neutrality condition at $T=150 \mathrm{MeV}$ (full) and $T=200$ $\mathrm{MeV}$ (empty). The idea is to simulate lower order fluctuations at imaginary $\mu_{B}$ and use them in a combined fit whose coefficients at $\mu_{B}=0$ are the higher order fluctuations. The formulas used for the combined fit of $\chi_{1}, \ldots \chi_{4}$ are

$$
\chi_{1}^{B}\left(\hat{\mu}_{B}\right)=2 c_{2} \hat{\mu}_{B}+4 c_{4} \hat{\mu}_{B}^{3}+6 c_{6} \hat{\mu}_{B}^{5}+\frac{4 !}{7 !} c_{4} \varepsilon_{1} \hat{\mu}_{B}^{7}+\frac{4 !}{9 !} c_{4} \varepsilon_{2} \hat{\mu}_{B}^{9}
$$




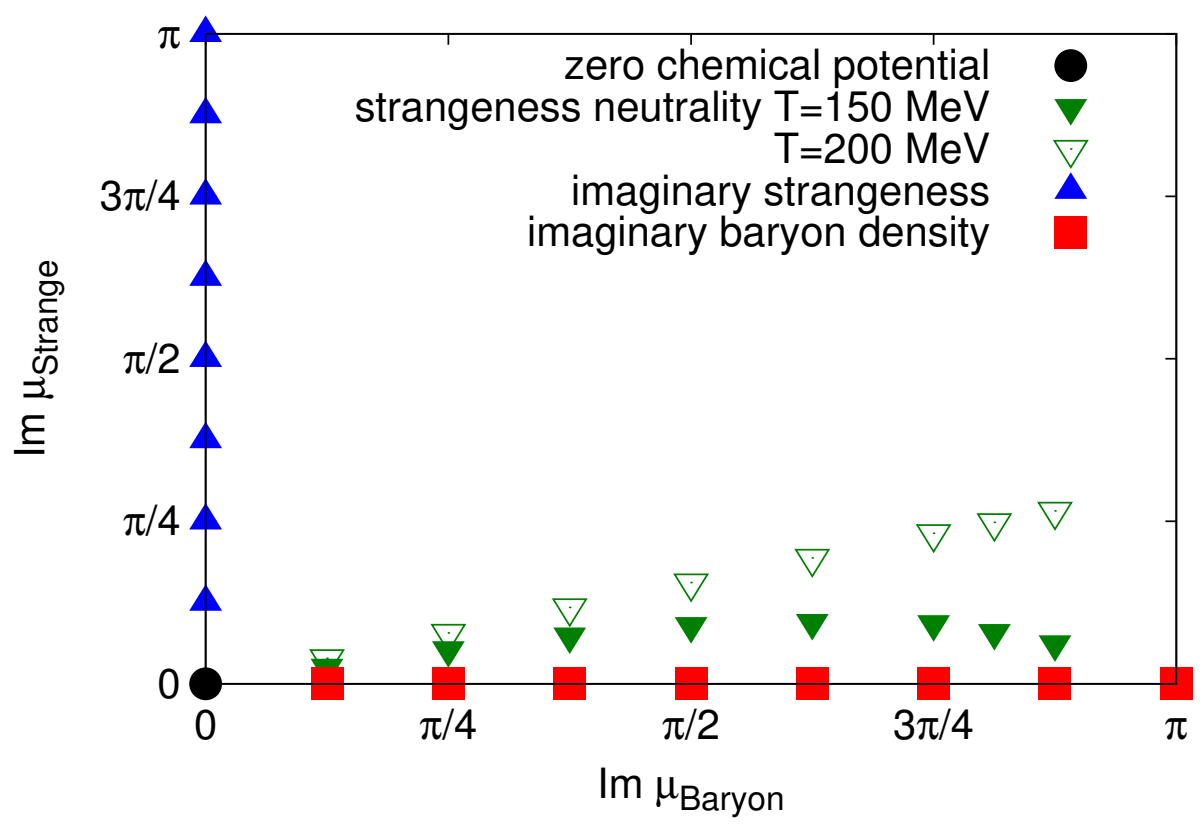

Figure 2: Simulation landscape in the imaginary $\mu_{B} / T-\mu_{S} / T$ plane of QCD. The QCD observables are periodic in the imaginary chemical potentials, thus only the range $(0 \ldots \pi) \times(0 \ldots \pi)$ has to be explored.

$$
\begin{aligned}
& \chi_{2}^{B}\left(\hat{\mu}_{B}\right)=2 c_{2}+12 c_{4} \hat{\mu}_{B}^{2}+30 c_{6} \hat{\mu}_{B}^{4}+\frac{4 !}{6 !} c_{4} \varepsilon_{1} \hat{\mu}_{B}^{6}+\frac{4 !}{8 !} c_{4} \varepsilon_{2} \hat{\mu}_{B}^{8} \\
& \chi_{3}^{B}\left(\hat{\mu}_{B}\right)=24 c_{4} \hat{\mu}_{B}+120 c_{6} \hat{\mu}_{B}^{3}+\frac{4 !}{5 !} c_{4} \varepsilon_{1} \hat{\mu}_{B}^{5}+\frac{4 !}{7 !} c_{4} \varepsilon_{2} \hat{\mu}_{B}^{7} \\
& \chi_{4}^{B}\left(\hat{\mu}_{B}\right)=24 c_{4}+360 c_{6} \hat{\mu}_{B}^{2}+c_{4} \varepsilon_{1} \hat{\mu}_{B}^{4}+\frac{4 !}{6 !} c_{4} \varepsilon_{2} \hat{\mu}_{B}^{6}
\end{aligned}
$$

where $\varepsilon_{1}$ and $\varepsilon_{2}$ are drawn randomly from a normal distribution with mean -1.25 and variance 2.75 . The authors of Ref. [44] perform a correlated fit for the four measured observables, thus obtaining the values of $c_{2}, c_{4}$ and $c_{6}$ for each temperature, and the corresponding $\chi_{2}^{B}, \chi_{4}^{B}$ and $\chi_{6}^{B}$ (notice that $\left.n ! c_{n}=\chi_{n}^{B}\right)$. These results are shown in Fig. 3, together with an estimate of $\chi_{8}^{B}$.

With the coefficients we have today we are able to cover the phase diagram for $\mu_{B} / T<2.5$.

I would like to mention two approaches, which allow us to bring the EoS closer to the experimental needs. The first one uses the lattice EoS up to order $\left(\mu_{B} / T\right)^{4}$ and introduces a critical point in the 3D Ising model universality class for QCD [45]. This family of equations of states will be useful to test the effect of the critical point on hydrodynamics simulations, to be compared to the experimental data from the second Beam Energy Scan. The other approach starts with a fugacity expansion for the baryonic density, motivated by the HRG model formula (2.1), and postulates all higher order coefficients to follow this behavior. This approach does not admit a critical point, but allows to predict the behavior of all Taylor expansion coefficients [46].

\section{QCD phase diagram}

In 2006, lattice QCD simulations showed that the QCD transition at $\mu_{B}=0$ is an analytical crossover [1]. The transition temperature is known since a few years [1, 47, 48, 49], with differ- 

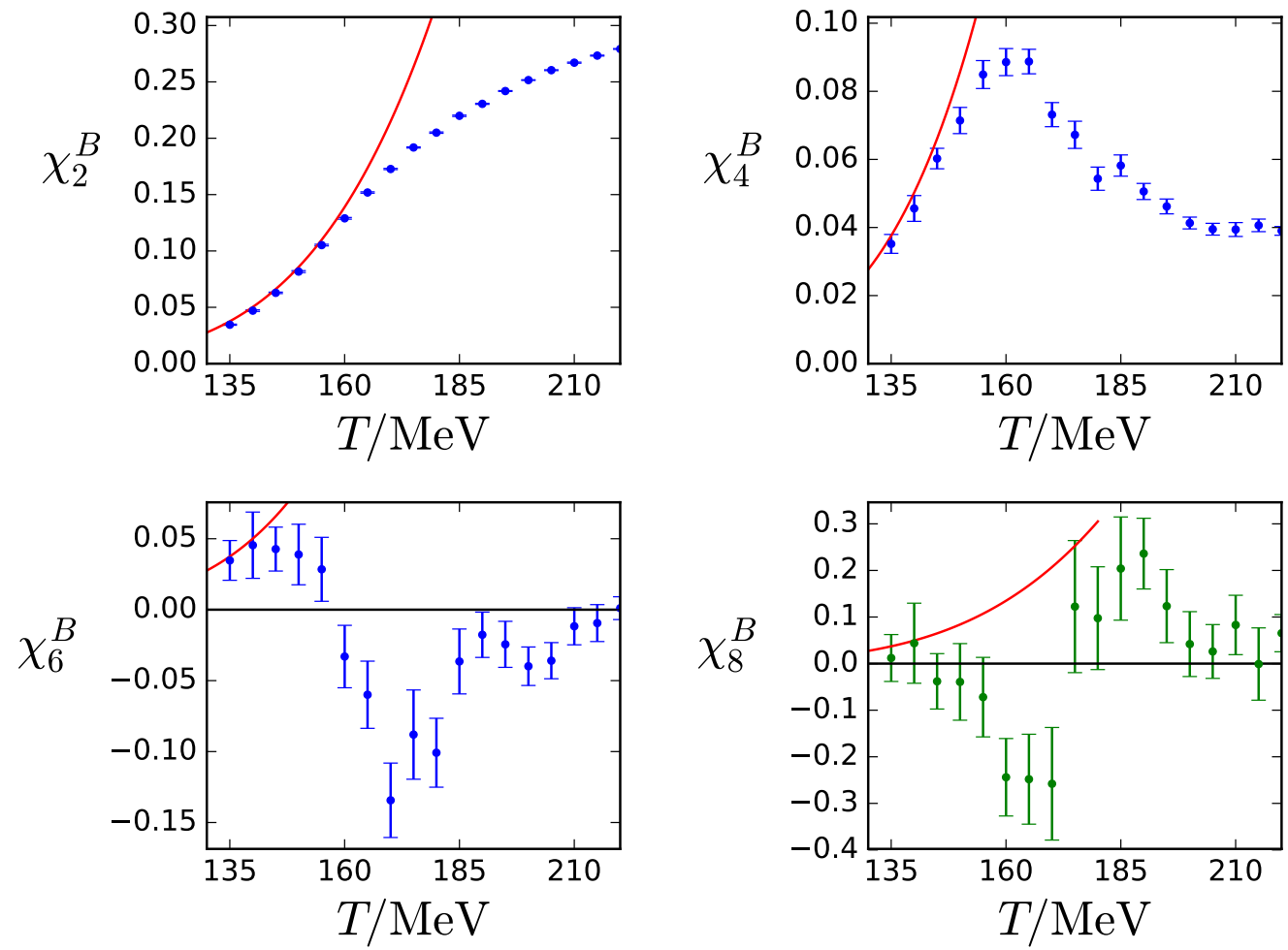

Figure 3: Results for $\chi_{2}^{B}, \chi_{4}^{B}, \chi_{6}^{B}$ and an estimate for $\chi_{8}^{B}$ as functions of the temperature, obtained from the single-temperature analysis. We plot $\chi_{8}^{B}$ in green to point out that its determination is guided by a prior. The red curve in each panel corresponds to the Hadron Resonance Gas (HRG) model result.

ent observables leading to slightly different results and defining the width of the crossover. The HotQCD collaboration has recently updated their value for the transition temperature based on chiral observables, considerably shrinking the error-bar [50]. The quoted value is $T_{c}=156.5 \pm 1.5$ $\mathrm{MeV}$.

Following the transition temperature location at finite chemical potential, one can define the curvature $\kappa$ of the QCD phase diagram

$$
\frac{T_{c}\left(\mu_{B}\right)}{T_{c}\left(\mu_{B}=0\right)}=1-\kappa\left(\frac{\mu_{B}}{T_{c}\left(\mu_{B}=0\right)}\right)^{2}-\lambda\left(\frac{\mu_{B}}{T_{c}\left(\mu_{B}=0\right)}\right)^{4} .
$$

Several results for $\kappa$ exist in the literature; they differ by approach and choices of strangeness and electric charge chemical potentials. A compilation of results is shown in Fig. 4.

The Taylor expansion of thermodynamic observables will break down if there is a critical point on the QCD phase diagram. The position of the critical point would then be identified with the radius of convergence of the Taylor series, provided that there is no other singularity in the imaginary $\mu_{B}$ plane, closer to $\mu_{B}=0$. The radius of convergence can be obtained from the ratio of subsequent coefficients in the Taylor expansion e.g. of the pressure or its derivatives. For the Taylor expansion of $\chi_{2}^{B}$, it is defined as follows

$$
r_{2 n}^{\chi}=\left|\frac{2 n(2 n-1) \chi_{2 n}^{B}}{\chi_{2 n+2}^{B}}\right|^{1 / 2} .
$$




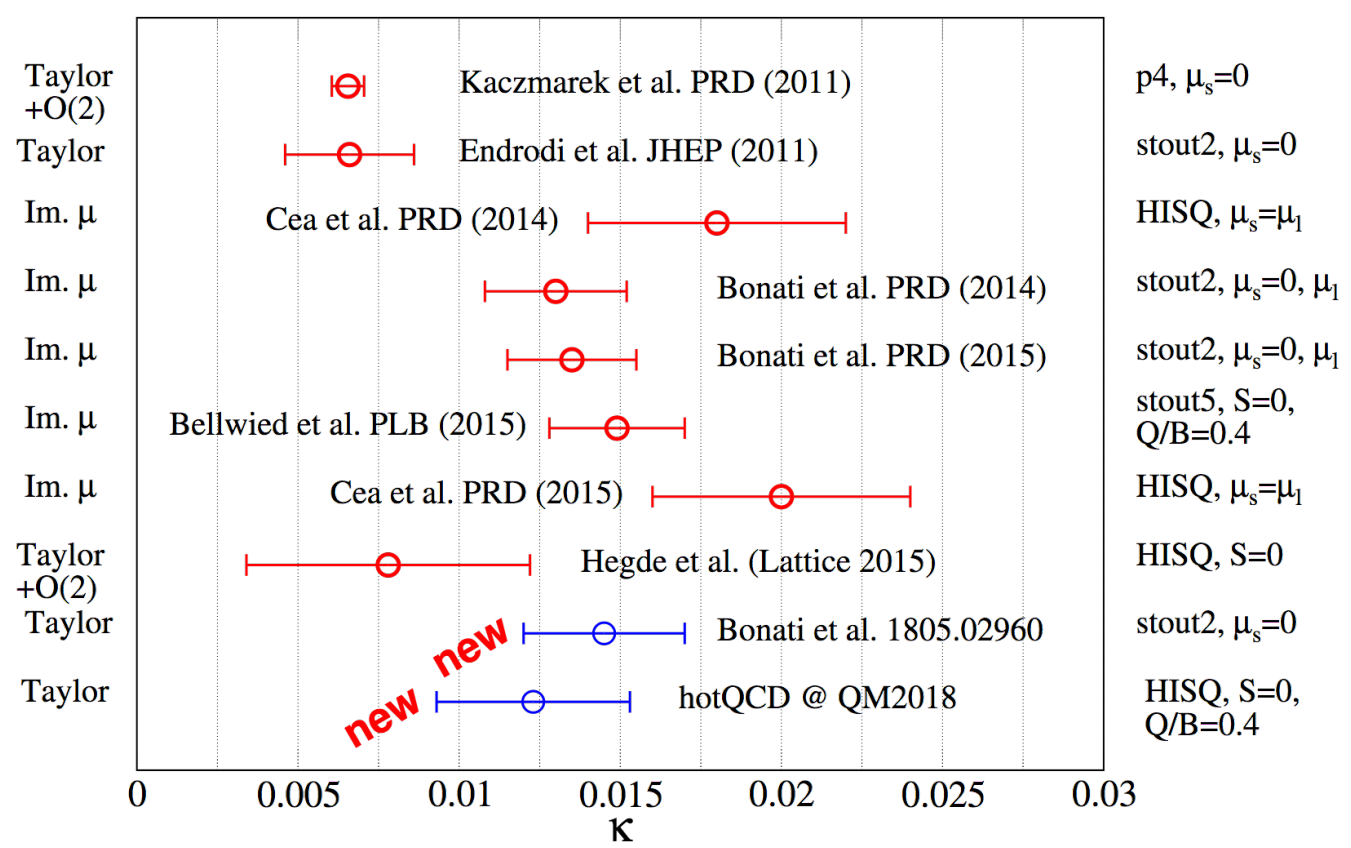

Figure 4: (From Ref. [51]) Compilation of results for the curvature of the phase diagram of QCD, obtained with different methods, different actions and different conditions on $\mu_{S}$ and $\mu_{Q}[21,52,53,54,55,56,57$, $58,59]$.

Strictly speaking, $r_{2 n}^{\chi}$ converges for $n \rightarrow \infty$. The authors of Ref. [60] re-did the analysis on the $N_{t}=4$ lattices with unimproved action by Fodor and Katz [31] with statistics increased by a factor 50 , and found the same critical point previously quoted. They found that the radius of convergence in that case seems to converge to the right value with just a few coefficients, probably due to the fact that the critical point is relatively close to $\mu_{B}=0$. For QCD on finer lattices, the most up-to-date estimate for the radius of convergence is from Refs. [42] and [43]. In the range $135 \mathrm{MeV} \leq T \leq$ $155 \mathrm{MeV}$ a critical point at $\mu_{B} / T<2$ is highly disfavored.

\section{Fluctuations of conserved charges}

Fluctuations of conserved charges allow us to characterize the chemical freeze-out, the moment in the evolution of a heavy ion collision at which all inelastic collisions between hadrons cease: the chemical composition of the system is fixed at this point. The cumulants of the experimental event-by-event distribution of a conserved charge are fluctuation observables which we can calculate on the lattice, and which are defined as

$$
\chi_{\text {lmn }}^{B S Q}=\frac{\partial^{l+m+n} p / T^{4}}{\partial\left(\mu_{B} / T\right)^{l} \partial\left(\mu_{S} / T\right)^{m} \partial\left(\mu_{Q} / T\right)^{n}}
$$

These quantities are fixed at the chemical freeze-out; the lattice curves are functions of the temperature, chemical potential and volume. The volume factor cancels out by taking ratios:

$$
\begin{array}{rlrl}
M / \sigma^{2} & =\chi_{1} / \chi_{2} & S \sigma & =\chi_{3} / \chi_{2} \\
S \sigma^{3} / M & =\chi_{3} / \chi_{1} & \kappa \sigma^{2} & =\chi_{4} / \chi_{2},
\end{array}
$$


where $M, \sigma, S, \kappa$ are the mean, variance, skewness and kurtosis of the experimental net-charge distribution, respectively. By comparing the experimental value to the lattice curves we can obtain the temperature and chemical potential at the chemical freeze-out $[61,62,63]$.

The STAR collaboration published results for the net-proton [64] and net-charge [65] fluctuations at different collision energies. Possible experimental sources of non-thermal fluctuations are corrected for in the STAR data analysis: the centrality-bin-width correction method minimizes effects due to volume variation because of finite centrality bin width (such effects have been studied e.g. in $[66,67,68])$; the moments are corrected for the finite reconstruction efficiency based on binomial probability distribution [69]. A $p_{T}$-dependent efficiency correction method [70] has been recently implemented; multiplicity-dependent and non-binomial efficiency corrections have also been studied [71], as well as the effect of baryon number conservation on the cumulants of net-proton distribution [72].

Final-state interactions in the hadronic phase and non-equilibrium effects might become relevant and affect fluctuations [73, 74, 75, 76, 77, 78, 79]; a fundamental check in favor of the equilibrium scenario is e.g. the consistency between the freeze-out parameters yielded by different quantum numbers, like electric charge and baryon number.

One more caveat is in order, since experimentally only the net-proton multiplicity distribution is measured, as opposed to the lattice net-baryon number fluctuations. It was shown that, once the effects of resonance feed-down and isospin randomization are taken into account [80,81], the net-proton and net-baryon number fluctuations are numerically very similar, at least in the case of low-order fluctuations [82].

In 2014 it was shown that two independent analyses based on fluctuations of baryon number and electric charge give consistent results for the freeze-out parameters [83]. More recently, a combined fit of the ratio of first-to-second order fluctuations for these two charges has been performed: since their experimental and theoretical uncertainties are smaller, the freeze-out temperature and chemical potential can be determined with a higher precision. The results of this analysis are shown in the left panel of Fig. 5. The colored lines are the trajectories in the $\left(T, \mu_{B}\right)$ plane which satisfy the experimental value: the points where they cross yield the desired freeze-out $T$ and $\mu_{B}$. Also shown in the figure are the isentropic expansion trajectories that the system in a heavy-ion collision would follow in the case of strictly zero viscosity; the red shaded area is the crossover transition region in the QCD phase diagram [56].

Experimentally, it is very difficult to measure strangeness fluctuations. The STAR collaboration has recently presented results for net-kaon fluctuations [88]. On the lattice it is possible to calculate fluctuations of conserved charges: the issue of isolating a single particle contribution is not trivial. For example, as shown in Eq. (2.1), in the hadronic phase it is possible to separate the contribution to the pressure of particles according to their quantum numbers: non- strange mesons, non-strange baryons, mesons with strangeness 1 , baryons with strangeness 1,2 , and 3 . Following this idea, and taking only the derivatives of the contribution of mesons with strangeness one, one can obtain the fluctuations of mesons with strangeness one. In a heavy ion collision there are two sources of mesons: the primordial ones, which are formed at hadronization, and the ones coming from resonance decays. The HRG model can provide both. In Ref [89] $\chi_{2} / \chi_{1}$ for kaons has been calculated in the HRG model in two ways, just by means of the Boltzmann approximation using the idea illustrated above, and by calculating the full contribution including primordial kaons and de- 

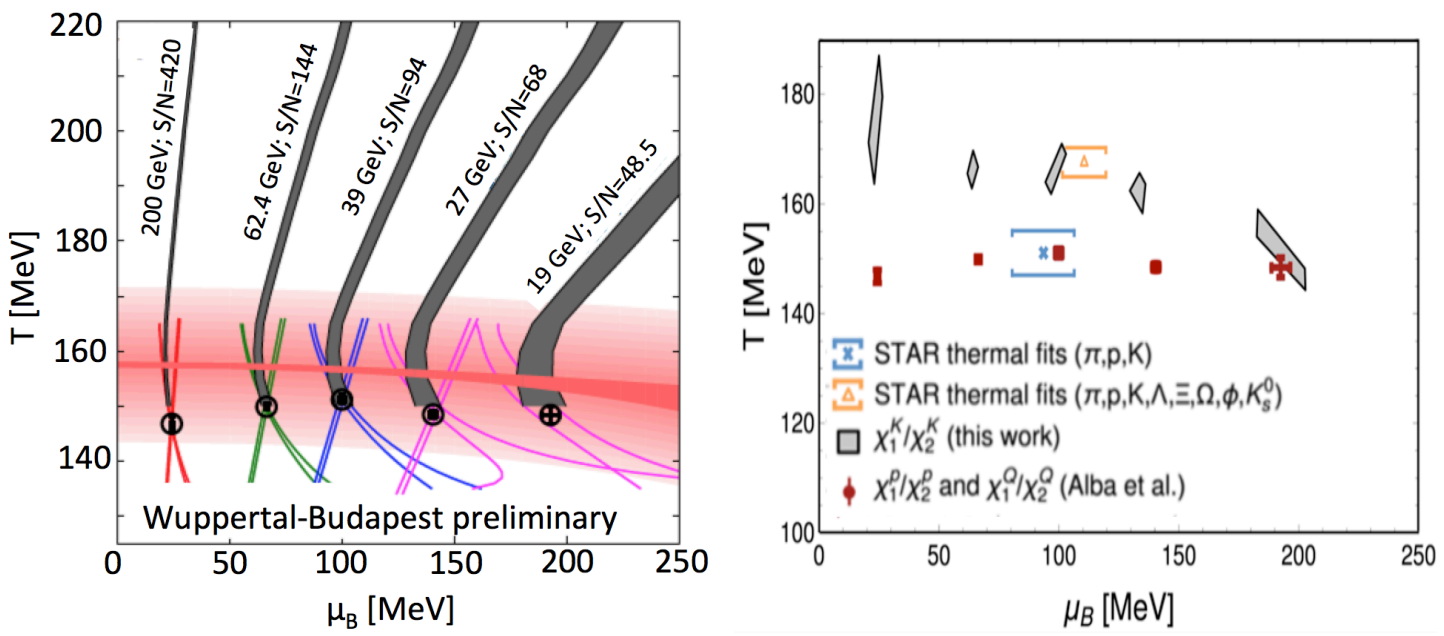

Figure 5: Left: Preliminary results of the WB collaboration [84, 85]. The colored lines are the contours at constant mean/variance ratios of the net electric charge from lattice simulations. The contours that correspond to STAR data intersect in the freeze-out points of Ref. [86]. The red band is the QCD phase diagram from Ref. [56]. Also shown are the isentropic contours that match the chemical freeze-out data. Right: Freeze-out parameters across the highest five energies from the Beam Energy Scan. The red points were obtained from the combined fit of $\chi_{1}^{p} / \chi_{2}^{p}$ and $\chi_{1}^{Q} / \chi_{2}^{Q}$ [86], while the gray bands are obtained from the fit of $\chi_{1}^{K} / \chi_{2}^{K}$ in this work. Also shown are the freeze-out parameters obtained by the STAR collaboration at $\sqrt{s}=39 \mathrm{GeV}$ [87] from thermal fits to all measured ground-state yields (orange triangle) and only to protons, pions and kaons (blue $\mathrm{x}$-shaped symbol).

cays. Since the two curves are very close to each other for all values of $T$ and $\mu_{B}$, it was concluded that it is possible to isolate the net-kaon $\chi_{2} / \chi_{1}$ on the lattice. It will be interesting to see whether the lattice QCD results for this observables confirm the HRG model analysis of kaons fluctuations, which led to a higher freeze-out temperature for kaons compared to the light particles [90]. The results of this analysis are shown in the right panel of Fig. 5.

Fluctuations can be used to study criticality, as they are expected to diverge with powers of the correlation length near the critical point $[19,91,92]$. Besides, fourth-order fluctuations are expected to be non-monotonic near the critical point [93, 94].

Recent results by the HotQCD collaboration showed the baryon number variance and the disconnected chiral susceptibility, extrapolated to finite $\mu_{B}$ along the crossover line. Both are expected to diverge at the critical point, but none of them shows any signs of criticality up to $\mu_{B}=250 \mathrm{MeV}$ [95].

As for the higher order fluctuations, $\chi_{3} / \chi_{1}$ and $\chi_{4} / \chi_{2}$ can be expanded in Taylor series in powers of $\mu_{B} / T$. The HotQCD collaboration pointed out that the Taylor expansion coefficients for $\chi_{4} / \chi_{2}$ is three times larger than the one for $\chi_{3} / \chi_{1}$, a trend which seems to be confirmed by the experimental data. However, recently it was pointed out in Ref. [96] that it is possible to explain the same trend in the data in terms of baryon number conservation and volume fluctuation effects. Results from the WB collaboration for $\chi_{3} / \chi_{1}$ and $\chi_{4} / \chi_{2}$ are consistent with these findings [44]. The extrapolation of these quantities to finite $\mu_{B}$ is shown in the two panels of Fig. 6. 

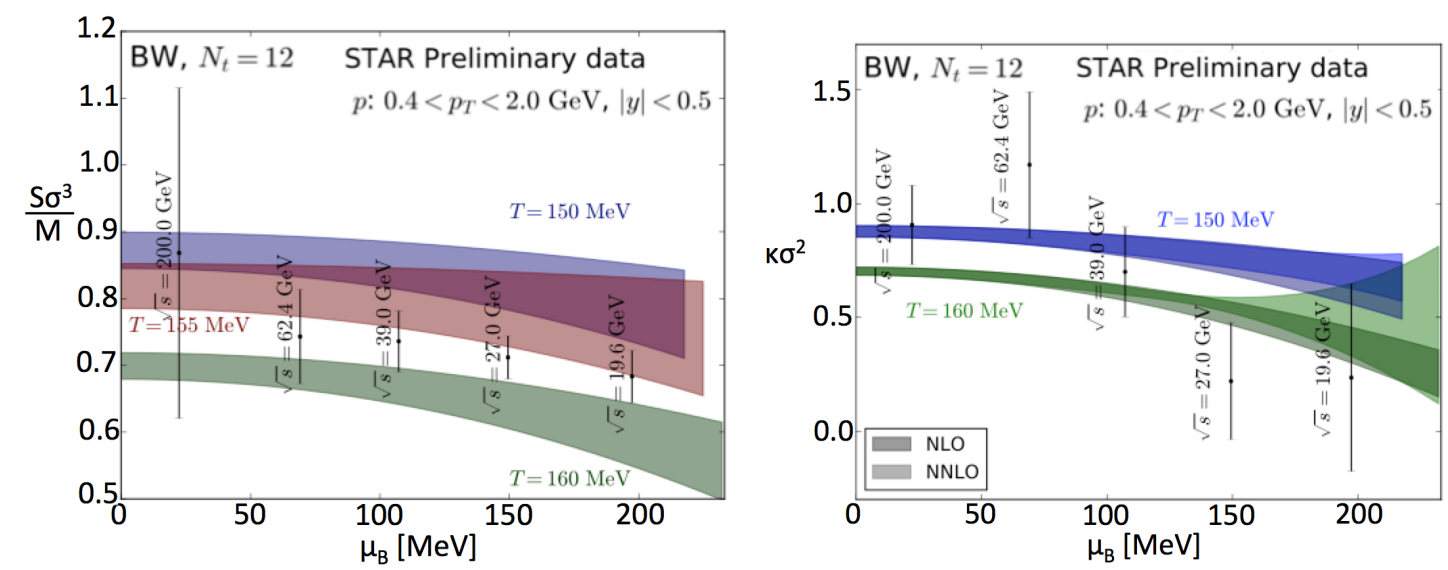

Figure 6: $S_{B} \sigma_{B}^{3} / M_{B}$ (left panel) and $\kappa_{B} \sigma_{B}^{2}$ (right panel) extrapolated to finite chemical potential. The left panel is extrapolated up to $\mathscr{O}\left(\hat{\mu}_{B}^{2}\right)$. In the right panel, the darker bands correspond to the extrapolation up to $\mathscr{O}\left(\hat{\mu}_{B}^{2}\right)$, whereas the lighter bands also include the $\mathscr{O}\left(\hat{\mu}_{B}^{4}\right)$ term.

\section{Conclusions}

The forthcoming experimental program, dedicated to the study of finite-density QCD, needs the theoretical support from lattice QCD simulations. This contribution collects an extensive compilation of results on equation of state, phase diagram and fluctuations of conserved charges. So far we have no indication of a critical point from lattice QCD. This is a very exciting time for the study of strongly interacting matter at finite density, and hopefully the joint effort of theory and experiment can lead to a map of the whole phase diagram of QCD relatively soon.

\section{Acknowledgements}

The author acknowledges useful discussions with her colleagues and collaborators Paolo Alba, Rene Bellwied, Szabolcs Borsanyi, Zoltan Fodor, Jana Günther, Sandor Katz, Volker Koch, Valentina Mantovani-Sarti, Jorge Noronha, Jacquelyn Noronha-Hostler, Paolo Parotto, Attila Pasztor, Israel Portillo, Kalman Szabo. This material is based upon work supported by the National Science Foundation under grants no. PHY-1654219 and OAC-1531814 and by the U.S. Department of Energy, Office of Science, Office of Nuclear Physics, within the framework of the Beam Energy Scan Theory (BEST) Topical Collaboration. The author also acknowledges the use of the Maxwell Cluster and the advanced support from the Center of Advanced Computing and Data Systems at the University of Houston.

\section{References}

[1] Aoki Y, Endrodi G, Fodor Z, Katz S D and Szabo K K 2006 Nature 443 675-678 (Preprint hep-lat/0611014)

[2] Jeon S and Heinz U 2015 Int. J. Mod. Phys. E24 1530010 (Preprint 1503.03931 )

[3] Dashen R, Ma S K and Bernstein H J 1969 Phys. Rev. 187 345-370

[4] Venugopalan R and Prakash M 1992 Nucl. Phys. A546 718-760 
[5] Bazavov A et al. 2014 Phys. Rev. Lett. 113072001 (Preprint 1404 . 6511)

[6] Alba P et al. 2017 (Preprint 1702.01113)

[7] Aoki Y, Fodor Z, Katz S D and Szabo K K 2006 JHEP 01089 (Preprint hep-lat / 0510084 )

[8] Borsanyi S, Endrodi G, Fodor Z, Jakovac A, Katz S D, Krieg S, Ratti C and Szabo K K 2010 JHEP 11077 (Preprint 1007.2580 )

[9] Borsanyi S, Fodor Z, Hoelbling C, Katz S D, Krieg S and Szabo K K 2014 Phys. Lett. B730 99-104 (Preprint 1309.5258 )

[10] Borsanyi S et al. 2016 Nature 539 69-71 (Preprint 1606.07494 )

[11] Alba P, Sarti V M, Noronha J, Noronha-Hostler J, Parotto P, Vazquez I P and Ratti C 2017 (Preprint $1711.05207)$

[12] Bazavov A et al. (HotQCD) 2014 Phys. Rev. D90 094503 (Preprint 1407 . 6387)

[13] Follana E, Mason Q, Davies C, Hornbostel K, Lepage G P, Shigemitsu J, Trottier H and Wong K (HPQCD, UKQCD) 2007 Phys. Rev. D75 054502 (Preprint hep-lat / 0610092 )

[14] Pratt S, Sangaline E, Sorensen P and Wang H 2015 Phys. Rev. Lett. 114202301 (Preprint $1501.04042)$

[15] Bernhard J E, Moreland J S, Bass S A, Liu J and Heinz U 2016 Phys. Rev. C94 024907 (Preprint $1605.03954)$

[16] Auvinen J, Karpenko I, Bernhard J E and Bass S A 2017 (Preprint 1706.03666)

[17] Allton C R, Ejiri S, Hands S J, Kaczmarek O, Karsch F, Laermann E, Schmidt C and Scorzato L 2002 Phys. Rev. D66 074507 (Preprint hep-lat/ 0204010 )

[18] Allton C R, Doring M, Ejiri S, Hands S J, Kaczmarek O, Karsch F, Laermann E and Redlich K 2005 Phys. Rev. D71 054508 (Preprint hep-lat/ 0501030 )

[19] Gavai R V and Gupta S 2008 Phys. Rev. D78 114503 (Preprint 0806.2233 )

[20] Basak S et al. (MILC) 2008 PoS LATTICE2008 171 (Preprint 0910 . 0276)

[21] Kaczmarek O, Karsch F, Laermann E, Miao C, Mukherjee S, Petreczky P, Schmidt C, Soeldner W and Unger W 2011 Phys. Rev. D83 014504 (Preprint 1011.3130)

[22] de Forcrand P and Philipsen O 2002 Nucl. Phys. B642 290-306 (Preprint hep-lat/ 0205016 )

[23] D'Elia M and Lombardo M P 2003 Phys. Rev. D67 014505 (Preprint hep-lat / 0209146 )

[24] Wu L K, Luo X Q and Chen H S 2007 Phys. Rev. D76 034505 (Preprint hep-lat / 0611035 )

[25] D’Elia M, Di Renzo F and Lombardo M P 2007 Phys. Rev. D76 114509 (Preprint 0705.3814 )

[26] Conradi S and D’Elia M 2007 Phys. Rev. D76 074501 (Preprint 0707 . 1987)

[27] de Forcrand P and Philipsen O 2008 JHEP 11012 (Preprint 0808.1096 )

[28] D’Elia M and Sanfilippo F 2009 Phys. Rev. D80 014502 (Preprint 0904.1400 )

[29] Moscicki J T, Wos M, Lamanna M, de Forcrand P and Philipsen O 2010 Comput. Phys. Commun. 181 1715-1726 (Preprint 0911.5682 )

[30] Fodor Z and Katz S D 2002 Phys. Lett. B534 87-92 (Preprint hep-lat/ 0104001 )

[31] Fodor Z and Katz S D 2002 JHEP 03014 (Preprint hep-lat/ 0106002 ) 
[32] Csikor F, Egri G I, Fodor Z, Katz S D, Szabo K K and Toth A I 2004 JHEP 05046 (Preprint hep-lat/0401016)

[33] Fodor Z and Katz S D 2004 JHEP 04050 (Preprint hep-lat / 0402006 )

[34] Alexandru A, Faber M, Horvath I and Liu K F 2005 Phys. Rev. D72 114513 (Preprint hep-lat/0507020)

[35] Kratochvila S and de Forcrand P 2006 PoS LAT2005 167 (Preprint hep-lat/ 0509143 )

[36] Ejiri S 2008 Phys. Rev. D78 074507 (Preprint 0804 . 3227)

[37] Fodor Z, Katz S D and Schmidt C 2007 JHEP 03121 (Preprint hep-lat/ 0701022 )

[38] Alexandru A, Gattringer C, Schadler H P, Splittorff K and Verbaarschot J J M 2015 Phys. Rev. D91 074501 (Preprint 1411.4143 )

[39] Borsanyi S, Endrodi G, Fodor Z, Katz S D, Krieg S, Ratti C and Szabo K K 2012 JHEP 08053 (Preprint 1204.6710 )

[40] Hegde P (BNL-Bielefeld-CCNU) 2014 Nucl. Phys. A931 851-855 (Preprint 1408 . 6305)

[41] Gunther J, Bellwied R, Borsanyi S, Fodor Z, Katz S D, Pasztor A and Ratti C 2017 EPJ Web Conf. 13707008 (Preprint 1607.02493 )

[42] Bazavov A et al. 2017 Phys. Rev. D95 054504 (Preprint 1701.04325 )

[43] D’Elia M, Gagliardi G and Sanfilippo F 2017 Phys. Rev. D95 094503 (Preprint 1611.08285 )

[44] Borsanyi S, Fodor Z, Guenther J N, Katz S K, Szabó K K, Pasztor A, Portillo I and Ratti C 2018 (Preprint 1805.04445)

[45] Parotto P, Bluhm M, Mroczek D, Nahrgang M, Noronha-Hostler J, Rajagopal K, Ratti C, Schäfer T and Stephanov M 2018 (Preprint 1805.05249 )

[46] Vovchenko V, Steinheimer J, Philipsen O and Stoecker H 2018 Phys. Rev. D97 114030 (Preprint $1711.01261)$

[47] Aoki Y, Borsanyi S, Durr S, Fodor Z, Katz S D, Krieg S and Szabo K K 2009 JHEP 06088 (Preprint $0903.4155)$

[48] Borsanyi S, Fodor Z, Hoelbling C, Katz S D, Krieg S, Ratti C and Szabo K K (Wuppertal-Budapest) 2010 JHEP 09073 (Preprint 1005 . 3508)

[49] Bhattacharya T et al. 2014 Phys. Rev. Lett. 113082001 (Preprint 1402.5175 )

[50] Ding H T, Hegde P, Karsch F, Lahiri A, Li S T, Mukherjee S and Petreczky P 2018 Chiral phase transition of (2+1)-flavor QCD 27th International Conference on Ultrarelativistic Nucleus-Nucleus Collisions (Quark Matter 2018) Venice, Italy, May 14-19, 2018 (Preprint 1807.05727 )

[51] Bonati C, D’Elia M, Negro F, Sanfilippo F and Zambello K 2018 (Preprint 1807.10026 )

[52] Endrodi G, Fodor Z, Katz S D and Szabo K K 2011 JHEP 04001 (Preprint 1102 . 1356)

[53] Cea P, Cosmai L and Papa A 2014 Phys. Rev. D89 074512 (Preprint 1403.0821 )

[54] Bonati C, D’Elia M, Mariti M, Mesiti M, Negro F and Sanfilippo F 2014 Phys. Rev. D90 114025 (Preprint 1410.5758 )

[55] Bonati C, D’Elia M, Mariti M, Mesiti M, Negro F and Sanfilippo F 2015 Phys. Rev. D92 054503 (Preprint 1507.03571) 
[56] Bellwied R, Borsanyi S, Fodor Z, Günther J, Katz S D, Ratti C and Szabo K K 2015 Phys. Lett. B751 559-564 (Preprint 1507.07510)

[57] Cea P, Cosmai L and Papa A 2016 Phys. Rev. D93 014507 (Preprint 1508 . 07599)

[58] Hegde P and Ding H T (Bielefeld-BNL-CCNU) 2016 PoS LATTICE2015 141 (Preprint $1511.03378)$

[59] Bonati C, D’Elia M, Negro F, Sanfilippo F and Zambello K 2018 Phys. Rev. D98 054510 (Preprint $1805.02960)$

[60] Fodor Z, Giordano M, Guenther J N, Kapas K, Katz S D, Pasztor A, Portillo I, Ratti C, Sexty D and Szabo K K 2018 Searching for a CEP signal with lattice QCD simulations 27th International Conference on Ultrarelativistic Nucleus-Nucleus Collisions (Quark Matter 2018) Venice, Italy, May 14-19, 2018 (Preprint 1807.09862 )

[61] Karsch F 2012 Central Eur. J. Phys. 10 1234-1237 (Preprint 1202.4173)

[62] Bazavov A et al. 2012 Phys. Rev. Lett. 109192302 (Preprint 1208.1220)

[63] Borsanyi S, Fodor Z, Katz S D, Krieg S, Ratti C and Szabo K K 2013 Phys. Rev. Lett. 111062005 (Preprint 1305.5161)

[64] Adamczyk L et al. (STAR) 2014 Phys. Rev. Lett. 112032302 (Preprint 1309.5681 )

[65] Adamczyk L et al. (STAR) 2014 Phys. Rev. Lett. 113092301 (Preprint 1402 . 1558)

[66] Skokov V, Friman B and Redlich K 2013 Phys. Rev. C88 034911 (Preprint 120 5 . 4756)

[67] Braun-Munzinger P, Rustamov A and Stachel J 2017 Nucl. Phys. A960 114-130 (Preprint 1612.00702 )

[68] Begun V and Mackowiak-Pawlowska M 2017 (Preprint 1705.01110 )

[69] Bzdak A and Koch V 2012 Phys. Rev. C86 044904 (Preprint 1206. 4286)

[70] Bzdak A and Koch V 2015 Phys. Rev. C91 027901 (Preprint 1312.4574)

[71] Bzdak A, Holzmann R and Koch V 2016 Phys. Rev. C94 064907 (Preprint 1603.09057 )

[72] Bzdak A, Koch V and Skokov V 2013 Phys. Rev. C87 014901 (Preprint 1203. 4529)

[73] Becattini F, Steinheimer J, Stock R and Bleicher M 2017 Phys. Lett. B764 241-246 (Preprint $1605.09694)$

[74] Rapp R and Shuryak E V 2001 Phys. Rev. Lett. 86 2980-2983 (Preprint hep-ph/ 0008326 )

[75] Rapp R and Shuryak E V 2002 Nucl. Phys. A698 587-590 (Preprint hep-ph / 0104006 )

[76] Rapp R 2002 Phys. Rev. C66 017901 (Preprint hep-ph/0204131)

[77] Steinheimer J, Aichelin J and Bleicher M 2013 Phys. Rev. Lett. 110042501 (Preprint 1203. 5302)

[78] Becattini F, Bleicher M, Kollegger T, Schuster T, Steinheimer J and Stock R 2013 Phys. Rev. Lett. 111 082302 (Preprint 1212.2431)

[79] Steinheimer J, Vovchenko V, Aichelin J, Bleicher M and Stöcker H 2016 (Preprint 1608.03737 )

[80] Kitazawa M and Asakawa M 2012 Phys. Rev. C85 021901 (Preprint 1107.2755 )

[81] Kitazawa M and Asakawa M 2012 Phys. Rev. C86 024904 [Erratum: Phys. Rev.C86,069902(2012)] (Preprint 1205.3292) 
[82] Nahrgang M, Bluhm M, Alba P, Bellwied R and Ratti C 2015 Eur. Phys. J. C75 573 (Preprint $1402.1238)$

[83] Borsanyi S, Fodor Z, Katz S D, Krieg S, Ratti C and Szabo K K 2014 Phys. Rev. Lett. 113052301 (Preprint 1403.4576)

[84] Ratti C 2016 Nucl. Phys. A956 51-58 (Preprint 1601.02367 )

[85] Ratti C 2018 Rept. Prog. Phys. 81084301 (Preprint 1804.07810 )

[86] Alba P, Alberico W, Bellwied R, Bluhm M, Mantovani Sarti V, Nahrgang M and Ratti C 2014 Phys. Lett. B738 305-310 (Preprint 1403.4903 )

[87] Adamczyk L et al. (STAR) 2017 Phys. Rev. C96 044904 (Preprint 1701 . 07065 )

[88] Adamczyk L et al. (STAR) 2018 Phys. Lett. B785 551-560 (Preprint 1709 . 00773)

[89] Noronha-Hostler J, Bellwied R, Gunther J, Parotto P, Pasztor A, Vazquez I P and Ratti C 2016 (Preprint 1607.02527 )

[90] Ratti C, Bellwied R, Noronha-Hostler J, Parotto P, Portillo Vazquez I and Stafford J M 2018 J. Phys. Conf. Ser. 1070 012003 (Preprint 1805.00088 )

[91] Stephanov M A, Rajagopal K and Shuryak E V 1999 Phys. Rev. D60 114028 (Preprint hep-ph/9903292)

[92] Cheng M et al. 2008 Phys. Rev. D77 014511 (Preprint 0710.0354 )

[93] Stephanov M A 2011 Phys. Rev. Lett. 107052301 (Preprint 1104.1627 )

[94] Ling B and Stephanov M A 2016 Phys. Rev. C93 034915 (Preprint 1512 . 09125)

[95] Steinbrecher P 2018 (Preprint 1807.05607)

[96] Braun-Munzinger P, Rustamov A and Stachel J 2018 (Preprint 1807. 08927) 